M. Ghorbel (Cergy-Pontoise and Paris)

T. Huillet (Cergy-Pontoise)

\title{
ON TWO FRAGMENTATION SCHEMES WITH ALGEBRAIC SPLITTING PROBABILITY
}

Abstract. Consider the following inhomogeneous fragmentation model: suppose an initial particle with mass $x_{0} \in(0,1)$ undergoes splitting into $b>1$ fragments of random sizes with some size-dependent probability $p\left(x_{0}\right)$. With probability $1-p\left(x_{0}\right)$, this particle is left unchanged forever. Iterate the splitting procedure on each sub-fragment if any, independently.

Two cases are considered: the stable and unstable case with $p\left(x_{0}\right)=x_{0}^{a}$ and $p\left(x_{0}\right)=1-x_{0}^{a}$ respectively, for some $a>0$. In the first (resp. second) case, since smaller fragments split with smaller (resp. larger) probability, one suspects some stabilization (resp. instability) of the fragmentation process.

Some statistical features are studied in each case, chiefly fragment size distribution, partition function, and the structure of the underlying random fragmentation tree.

1. Introduction. Suppose an initial particle with mass (or size) $x_{0} \in$ $(0,1)$ possibly undergoes splitting into $b$ sub-fragments with some sizedependent probability $p\left(x_{0}\right)$. If splitting occurs, the active parental mass is shared between its $b>1$ offspring at random, different laws of partition fitting different splitting processes. With complementary probability $\bar{p}\left(x_{0}\right):=1-p\left(x_{0}\right)$, splitting does not take place and the ancestral fragment remains inactive forever. The effect of this splitting/non-splitting feature of such a fragmentation model is to stabilize the global number of splitting events. Its inhomogeneous character is a consequence of the splitting probability depending on fragment sizes.

2000 Mathematics Subject Classification: Primary 60G57, 62E17; Secondary 60K99, 62E15, 62E20.

Key words and phrases: fragmentation models. 
This size-dependent splitting process is next iterated independently on first-generation sub-fragments if ever generated, with induced size-dependent fragmentation probability; the process is then iterated over subsequent generations.

In a first (stable) model investigated, the splitting probability $p\left(x_{0}\right)$ grows algebraically with fragment size at each step; specifically, $p\left(x_{0}\right)=x_{0}^{a}$ for some $a>0$. Since smaller fragments split with smaller probability, one suspects some stabilization of the fragmentation process. Some aspects of this problem were recently investigated in Huillet (2005); we recall some of the results and develop additional aspects of the stable splitting model.

In a second (unstable) model, the splitting probability $p\left(x_{0}\right)$ now decays algebraically with fragment sizes at each step; specifically, $p\left(x_{0}\right)=1-x_{0}^{a}$ for some $a>0$. Once formed, smaller fragments split with larger probability, producing new smaller sub-fragments themselves very likely to further split, and so forth; some instability of the fragmentation process is expected to be induced by such a reinforcement design, resulting from an increased breakability of small items.

The purpose of this note is to initiate a comparative study of some basic statistical features shared by both toy-models.

Roughly speaking, the physical image we have in mind is the following: suppose a collection of items with different (but bounded above) sizes is subject to an external source of random shocks or collisions. In the first stable model, items once hit will split: larger items being more likely to be hit, the splitting probability increases with size. In the second unstable model, all items are equally likely to be hit, regardless of their sizes. But splitting of an item is not automatic if once hit: due to intrinsic fragility, small items are more likely to be smashed than larger ones.

Inhomogeneous fragmentation processes presenting loose analogies with our model were considered by Brennan and Durrett $(1986,1987)$, in continuous time (see also Filippov, 1961). In their model, with probability 1, an initial particle with mass $x_{0}>0$ undergoes splitting into two uniform subfragments in random exponential time with size-dependent rate $x_{0}^{a}$. The process is iterated on subsequent fragments, independently. If $a>0$, the smaller the fragment size, the smaller its fragmentation rate; the process stabilizes in the sense that the empirical distribution function of rescaled fragment sizes converges to some random variable. If $a<0$, the smaller the fragment size, the larger its fragmentation rate; the process becomes unstable. In this unstable case, although mass is conserved, the number (and next the mass) of fragments in the fragmentation process explodes (vanishes) in finite time; see Filippov (1961) and Brennan and Durrett (1986, 1987). In Filippov's paper (p. 276), the unstable model was considered to be physically unrealistic. However, in the subsequent years, this model received considerable 
attention as it was responsible for the shattering transition (formation of dust), a phenomenon considered by Ziff as the counterpart of gelation phenomenon (infinite cluster formation) in coagulation processes. See McGrady and Ziff (1987) and Jeon (2002), for example. In contrast with our model, size-dependence of successive fragmentations appears here in the rates, not in the splitting probability.

Clearly, our unstable (with size-dependent splitting probability) process also enhances formation of dust but to a lesser extent: indeed, by giving an opportunity of not splitting, one expects the limiting coexistence of both macroscopic and microscopic elements in the fragmentation tree.

2. First-generation partition law. There are many ways to split $x_{0}$ into $b$ offspring fragments with random sizes. So we need to be more specific.

Start then with an initial interval of mass (or size) $x_{0} \in(0,1)$. With probability $p\left(x_{0}\right)$, this interval will split. If it splits, it splits into $b>1$ fragments with random sizes, say $\mathbf{U} x_{0}:=\left(U_{1} x_{0}, \ldots, U_{b} x_{0}\right)$. Here $\mathbf{U}:=\left(U_{1}, \ldots, U_{b}\right)$ is assumed to have exchangeable distribution, with $U \stackrel{d}{=} U_{1} \stackrel{d}{=} \cdots \stackrel{d}{=} U_{b}$. We assume that $U$ has density $\pi(u)>0$ on $(0,1)$. Further, we shall assume a strict mass-conservation property: $\sum_{k=1}^{b} U_{k}=1$, a.s.

More specifically, we shall restrict ourselves to the following first-generation partitioning law: fix $\theta>0$ and assume that $\mathbf{U}:=\left(U_{1}, \ldots, U_{b}\right)$ is distributed according to the symmetric Dirichlet- $D_{b}(\theta)$ density function on the simplex, that is to say,

$$
\pi\left(u_{1}, \ldots, u_{b}\right)=\frac{\Gamma(b \theta)}{\Gamma(\theta)^{b}} \prod_{k=1}^{b} u_{k}^{\theta-1} \cdot \delta_{\left(\sum_{k=1}^{b} u_{k}=1\right)} .
$$

Alternatively, $\mathbf{U}:=\left(U_{1}, \ldots, U_{b}\right)$ is characterized by its joint moment function

$$
\phi\left(q_{1}, \ldots, q_{b}\right):=\mathbb{E}\left[\prod_{k=1}^{b} U_{k}^{q_{k}}\right]=\frac{\Gamma(b \theta)}{\Gamma\left(b \theta+\sum_{k=1}^{b} q_{k}\right)} \prod_{k=1}^{b} \frac{\Gamma\left(\theta+q_{k}\right)}{\Gamma(\theta)} .
$$

In this case, $U_{k} \stackrel{d}{=} U, k=1, \ldots, b$, and the individual fractions are all identically distributed. Their common density $\pi(u)$ on the interval $(0,1)$ is the one of a $\operatorname{beta}(\theta,(b-1) \theta)$ random variable. Stated differently, the moment function of $U$ reads

$$
\phi(q):=\mathbb{E}\left(U^{q}\right)=\frac{\Gamma(b \theta)}{\Gamma(b \theta+q)} \frac{\Gamma(\theta+q)}{\Gamma(\theta)}, \quad q>-\theta,
$$

with mean $\mathbb{E}(U)=1 / b$ and variance $\sigma^{2}(U)=(b-1) / b^{2}(b \theta+1)$.

The parameter $\theta$ indicates how concentrated the distribution of $\mathbf{U}$ is around its mean $(1 / b, \ldots, 1 / b)$ : the larger $\theta$, the more concentrated the dis- 
tribution of $\mathbf{U}$ around the mean. The intermediate case $\theta=1$ corresponds to the usual uniform partition into $b$ fragments for which

$$
\phi(q)=\frac{(b-1) !}{(q+1)_{b-1}}, \quad q>-1,
$$

where $(q)_{b}:=q(q+1)(q+2) \cdots(q+b-1)$.

3. The stable fragmentation model. We first suppose that, with $x_{0} \in(0,1)$, the splitting probability is $p\left(x_{0}\right)=x_{0}^{a}, a>0$, increasing with initial fragment size $x_{0}$. In this model, smaller fragments, once produced, generate (with smaller probability) other fragments with smaller sizes with themselves a smaller probability to undergo further splitting and so on. One suspects stability in that the fragmentation process (tree) should terminate with probability 1 (respectively, should be finite).

We first derive the fragment size distribution and then study the induced tree structure.

3.1. Stable fragment size distribution. A particular realization of our fragmentation process is clearly a marked $b$-ary sub-tree of the full $b$-ary tree. At step $h \geq 1$ of the fragmentation process, the fragmentation sub-tree has height at most $h$. It has height exactly $h$ if there is still at least one active (possibly splitting) node at this step. Otherwise, it has height strictly less than $h$ as fragmentation stopped before $h$ and the tree has only inactive leaves.

We shall consider the random variable $X_{h}\left(x_{0}\right)$, defined to be the mass attached to a random leaf of a step- $h$ realization of the stable fragmentation process. By a random leaf, we mean the leaf reached in at most $h$ steps by choosing at each step one of the sub-fragments with probability $1 / b$ whenever the node is an active one, and stopping the sampling process otherwise; $X_{h}\left(x_{0}\right)$ is thus the terminal mass in a random path from the step- $h$ fragmentation sub-tree. Call it fragment size at step $h$.

Let now $U_{1}, \ldots, U_{h}, \ldots$ be a sequence of replicas of $U$. Introduce the random quantity $H\left(x_{0}\right)=\inf \left\{h \geq 1: B_{h}\left(x_{0}\right)=0\right\}-1$. Here $\left\{B_{h}\left(x_{0}\right): h \geq 1\right\}$ is a sequence of Bernoulli $\left(\left(U_{1} \cdots U_{h-1} x_{0}\right)^{a}\right)$ distributed random variables indicating if splitting took place or not at step $h ; \mathbb{P}\left(B_{h}\left(x_{0}\right)=1\right)=x_{0}^{a} \phi(a)^{h-1}$. Clearly, $H\left(x_{0}\right)$ represents the limiting height of a random path in the full fragmentation tree.

Using the renewal structure of the process, we easily obtain the recursive identity in distribution

$$
X_{h+1}\left(x_{0}\right) \stackrel{d}{=} x_{0} \bar{B}\left(x_{0}\right)+B\left(x_{0}\right) X_{h}^{\prime}\left(U x_{0}\right), \quad h \geq 0, \quad X_{0}\left(x_{0}\right)=x_{0} .
$$

Here $B\left(x_{0}\right)$ is a $\{0,1\}$-valued $\operatorname{Bernoulli}\left(x_{0}^{a}\right)$ random variable, taking the value 1 with splitting probability $p\left(x_{0}\right)=x_{0}^{a} ; \bar{B}\left(x_{0}\right):=1-B\left(x_{0}\right) ; X_{h}^{\prime}(\cdot) \stackrel{d}{=}$ 
$X_{h}(\cdot)$ is a statistical copy of $X_{h}(\cdot)$ and $\left(B\left(x_{0}\right), X_{h}^{\prime}\left(U x_{0}\right), U\right)$ are mutually independent random variables.

Let us briefly comment on the identity (3): if there is no splitting at step one (the event $\bar{B}\left(x_{0}\right)=1$ occurring with probability $\bar{p}\left(x_{0}\right)$ ) the random size $X_{h+1}\left(x_{0}\right)$ at step $h+1$ is just the size $x_{0}$ of the initial fragment that does not undergo further fragmentation. If splitting occurs at step one (the event $B\left(x_{0}\right)=1$ with probability $\left.p\left(x_{0}\right)\right), X_{h+1}\left(x_{0}\right)$ coincides in law with $X_{h}^{\prime}\left(U x_{0}\right)$ at step $h$, after rescaling $x_{0}$ properly by $U$. Since $H\left(x_{0}\right)<\infty$ a.s., the limiting fragment size $X\left(x_{0}\right):=X_{\infty}\left(x_{0}\right)$ exists as $h \uparrow \infty$. In Huillet (2005), the following result was obtained by iteration:

Proposition 1. As $h \uparrow \infty$, the limiting fragment size $X\left(x_{0}\right):=X_{\infty}\left(x_{0}\right)$ satisfies the distributional equality

$$
X\left(x_{0}\right) \stackrel{d}{=} x_{0} \bar{B}\left(x_{0}\right)+B\left(x_{0}\right) X^{\prime}\left(U x_{0}\right) .
$$

If $\Phi_{x_{0}}(q):=\mathbb{E}\left(X\left(x_{0}\right)^{q}\right)$ is its moment function, then for $q>q_{*}=-\theta$, we have

$$
\Phi_{x_{0}}(q)=x_{0}^{q}\left(1-\sum_{m \geq 1} a_{m}(q) x_{0}^{m a}\right)
$$

Here,

$$
\begin{aligned}
a_{1}(q) & =1-\phi(q), \\
a_{m}(q) & =a_{1}(q) \prod_{k=1}^{m-1} \phi(q+k a), \quad m \geq 2,
\end{aligned}
$$

and $\phi(q)$ is given by $(2)$.

Note that the series representation of $\Phi_{x_{0}}(q)$ is the unique $C^{\infty}$ solution of

$$
\Phi_{x_{0}}(q)=x_{0}^{q}\left(1-x_{0}^{a}\right)+x_{0}^{a} \int_{0}^{1} \Phi_{u x_{0}}(q) \pi(u) d u .
$$

Uniqueness for integral equations of the type (5) is indeed a consequence of Lemma 3 of Iserles and Liu (1997).

Remark. The law of $X\left(x_{0}\right)$ clearly has an atom at $x=x_{0}$ with mass $1-x_{0}^{a}$ when splitting does not take place. Observing that $\Phi_{x_{0}}(0)=1$, we have $\mathbb{P}\left(X\left(x_{0}\right)>0\right)=1$ : the height $H\left(x_{0}\right)$ of a random path being finite with probability 1 , the probability that $X\left(x_{0}\right)=0$ is null.

When $a \downarrow 0$, for $q>0, \Phi_{x_{0}}(q) \rightarrow 0$; this means that $X\left(x_{0}\right) \rightarrow 0$ in distribution and in probability: as splitting probability tends to 1 , the fragment size tends to zero. When $a \uparrow \infty$, the splitting probability tends to 0 and one may check that $\Phi_{x_{0}}(q) \sim x_{0}^{q}$ : the initial fragment is left unchanged forever. Finally, the range of $X\left(x_{0}\right)$ is $\left[0, x_{0}\right]$. 
ExAmple. Suppose $a=1 / 2, b=3$ and $\theta=1 / 2$. In this case, $U \stackrel{d}{\sim}$ $\operatorname{beta}(1 / 2,1)$ and $\phi(q)=(2 q+1)^{-1}$. So,

$$
a_{m}(q)=\frac{2 q}{\prod_{k=1}^{m}(2 q+k)} .
$$

Thus, $a_{m}:=a_{m}(1)=4 /(m+2)$ !, leading to the average fragment length

$$
x\left(x_{0}\right)=4+3 x_{0}+4 \sqrt{x_{0}}-4 e^{\sqrt{x_{0}}} \in\left(0, x_{0}\right) .
$$

3.2. Number of fragments in the stable fragmentation tree. Let $N_{h}\left(x_{0}\right)$ be the number of leaves, active or not, in the stable fragmentation tree at step $h$. Clearly, as $h \uparrow \infty$, the limiting number of leaves (fragments), starting with an interval of size $x_{0}$, is a well defined random variable; call it $N\left(x_{0}\right)$. Plainly, with $N_{k}(\cdot) \stackrel{d}{=} N(\cdot)$, it satisfies

$$
N\left(x_{0}\right) \stackrel{d}{=} \bar{B}\left(x_{0}\right)+B\left(x_{0}\right) \sum_{k=1}^{b} N_{k}\left(U_{k} x_{0}\right) .
$$

Let us first consider its expected value $m\left(x_{0}\right):=\mathbb{E}\left(N\left(x_{0}\right)\right)$. If $x_{0} \in(0,1)$, we have

$$
m\left(x_{0}\right)=1-x_{0}^{a}+b x_{0}^{a} \int_{0}^{1} m\left(u x_{0}\right) \pi(u) d u .
$$

Similarly, using Lemma 3 of Iserles and Liu (1997), by iteration, we obtain a result already stated in Huillet (2005):

Proposition 2.

(i) The series representation of $m\left(x_{0}\right)$,

$$
m\left(x_{0}\right)=1+\sum_{m \geq 1} b_{m} x_{0}^{m a},
$$

is the unique $C^{\infty}$ solution in $x_{0}$ of (6). The sequence $b_{m}, m \geq 1$, is defined by

$$
\begin{aligned}
b_{1} & =b-1, \\
b_{m} & =b_{1} b^{m-1} \prod_{k=1}^{m-1} \phi(k a), \quad m \geq 2 .
\end{aligned}
$$

(ii) For any $a>0$ and $x_{0} \in(0,1), m\left(x_{0}\right)<\infty$.

ExAmple. Let $b=3, \theta=1$ and $\phi(q)=(2 q+1)^{-1}$. Assuming $a=1 / 2$, we find

$$
b_{m}=2 \cdot 3^{m-1} \prod_{k=1}^{m-1} \frac{1}{k+1}=2 \frac{3^{m-1}}{m !} ;
$$

the expected number of leaves is $m\left(x_{0}\right)=\frac{1}{3}\left(2 e^{3 \sqrt{x_{0}}}+1\right)<\infty$. 
3.3. Partition function. Let $X_{i}\left(x_{0}\right)$ be the mass attached to fragment number $i, i=1, \ldots, N\left(x_{0}\right)$, in the stable limiting fragmentation tree. The system $\left(X_{i}\left(x_{0}\right) ; i=1, \ldots, N\left(x_{0}\right)\right)$ constitutes a random partition of $\mathbb{I}=[0,1]$. We shall study its random partition function.

Consider the random partition function

$$
Z_{x_{0}}(\beta):=\sum_{i=1}^{N\left(x_{0}\right)} X_{i}\left(x_{0}\right)^{\beta} .
$$

with $Z_{x_{0}}(1)=x_{0}, Z_{x_{0}}(0)=N\left(x_{0}\right)$. If $Z_{x_{0}, k}(\beta), k=1, \ldots, b$, are replicas of $Z_{x_{0}}(\beta)$, then $Z_{x_{0}}(\beta)$ solves

$$
Z_{x_{0}}(\beta) \stackrel{d}{=} x_{0}^{\beta} \bar{B}\left(x_{0}\right)+B\left(x_{0}\right) \sum_{k=1}^{b} Z_{U_{k} x_{0}, k}(\beta) .
$$

Let $z_{x_{0}}(\beta):=\mathbb{E}\left(Z_{x_{0}}(\beta)\right)$ be the first moment of $Z_{x_{0}}(\beta)$. Then

$$
z_{x_{0}}(\beta)=x_{0}^{\beta}\left(1-x_{0}^{a}\right)+b x_{0}^{a} \int_{0}^{1} z_{u x_{0}}(\beta) \pi(u) d u .
$$

We obtain the following result:

Proposition 3.

(i) When $\beta>\beta_{*}=-\theta, z_{x_{0}}(\beta)$ is well defined and

$$
z_{x_{0}}(\beta)=x_{0}^{\beta}\left(1-\sum_{m \geq 1} b_{m}(\beta) x_{0}^{m a}\right) \text {, }
$$

where

$$
\begin{aligned}
& b_{1}(\beta)=1-b \phi(\beta), \\
& b_{m}(\beta)=b_{1}(\beta) b^{m-1} \prod_{k=1}^{m-1} \phi(\beta+k a), \quad m \geq 2,
\end{aligned}
$$

is the unique $C^{\infty}$ solution in $x_{0}$ of $(8)$.

(ii) Let $\sigma(d x)$ be the structural (or occupation) measure of the partition system $\left(X_{i}\left(x_{0}\right) ; i=1, \ldots, N\left(x_{0}\right)\right)$, with

$$
\sigma(d x):=\mathbb{E} \sum_{i=1}^{N\left(x_{0}\right)} \mathbf{I}\left(X_{i}\left(x_{0}\right) \in d x\right) .
$$

Then

$$
\sigma(d x) / d x \underset{x \downarrow 0}{\sim} C_{a}\left(x_{0}\right) x^{-(1-\theta)}
$$


for the positive constant

$$
C_{a}\left(x_{0}\right)=b x_{0}^{a-\theta} \frac{\Gamma(b \theta)}{\Gamma((b-1) \theta) \Gamma(\theta)}\left(1+\sum_{m \geq 1}\left(b x_{0}^{a}\right)^{m} \prod_{k=1}^{m} \phi(-\theta+k a)\right) .
$$

Proof. (i) Looking for formal solutions of $z_{x_{0}}(\beta)$ of the form

$$
z_{x_{0}}(\beta)=x_{0}^{\beta}\left(1-\sum_{m \geq 1} b_{m}(\beta) x_{0}^{m a}\right),
$$

we obtain the coefficients

$$
\begin{aligned}
b_{1}(\beta) & =1-b \phi(\beta), \\
b_{m}(\beta) & =b b_{m-1}(\beta) \phi((m-1) a+\beta), \quad m \geq 2 .
\end{aligned}
$$

(ii) Under our assumption, the series with positive terms $\sum_{m \geq 1} b_{m}(\beta) x_{0}^{m a}$ is always convergent because

$$
\frac{b_{m+1}(\beta) x_{0}^{(m+1) a}}{b_{m}(\beta) x_{0}^{m a}}=b \phi(\beta+m a) x_{0}^{a} \underset{m \uparrow \infty}{\longrightarrow} 0 .
$$

As a result, $z_{x_{0}}(\beta)$ is absolutely convergent, hence convergent. This proves the following: let $\sigma(d x)$ be the structural measure of the system $\left(X_{i}\left(x_{0}\right)\right.$; $i \geq 1)$. It has Mellin transform

$$
z_{x_{0}}(\beta)=\int_{0}^{x_{0}} x^{\beta} \sigma(d x)
$$

It is easy to check that

$$
z_{x_{0}}(\beta)=x_{0}^{\beta}\left(1-b_{1}(\beta) x_{0}^{a}\left(1+\sum_{m \geq 1}\left(b x_{0}^{a}\right)^{m} \prod_{k=1}^{m} \phi(\beta+k a)\right)\right) .
$$

Now, it is clear that $\beta_{*}=-\theta$ is a dominant pole of $z_{x_{0}}(\beta)$, with $z_{x_{0}}(\beta) \sim_{\beta=-\theta}$ $C_{a}\left(x_{0}\right) /(\beta+\theta)$ and $C_{a}\left(x_{0}\right)$ given by (ii). Finally, from elementary singularity analysis we get

$$
\sigma(d x) / d x \underset{x \downarrow 0}{\sim} C_{a}\left(x_{0}\right) x^{-(1-\theta)} .
$$

Note that $\beta_{*}=-\theta<0$ is independent of $a$. When $\theta<1, \sigma(d x) / d x$ diverges at $x=0$, whereas if $\theta>1, \sigma(d x) / d x$ vanishes at $x=0$. When $\theta=1$, $\sigma(d x) / d x \sim_{x \downarrow 0} C_{a}\left(x_{0}\right)$.

REMARK. The behavior at $x=0$ of the occupation measure is an interesting information, for example for the following reason. Let $n_{-}(\varepsilon)=$ $\mathbb{E}\left[N_{-}(\varepsilon)\right]$, where $N_{-}(\varepsilon):=\sum_{i \geq 1} \mathbf{I}\left(X_{i}\left(x_{0}\right) \leq \varepsilon\right)$ counts the number of fragments whose size is less than $\varepsilon>0$. We clearly have

$$
n_{-}(\varepsilon)=\int_{0}^{\varepsilon} \sigma(d x) \underset{\varepsilon \downarrow 0}{\sim} \frac{C_{a}\left(x_{0}\right)}{\theta} \varepsilon^{\theta} .
$$


Similarly, let $x_{-}(\varepsilon)=\mathbb{E}\left[X_{-}(\varepsilon)\right]$, where $X_{-}(\varepsilon):=\sum_{i \geq 1} X_{i}\left(x_{0}\right) \mathbf{I}\left(X_{i}\left(x_{0}\right) \leq \varepsilon\right)$ is the contribution to the total mass of the fragments whose size is less than $\varepsilon$. We obtain

$$
x_{-}(\varepsilon)=\int_{0}^{\varepsilon} x \sigma(d x) \underset{\varepsilon \downarrow 0}{\sim} \frac{C_{a}\left(x_{0}\right)}{\theta+1} \varepsilon^{\theta+1} .
$$

4. Unstable fragmentation model. We now suppose that, for $x_{0} \in$ $(0,1)$, the splitting probability is $p\left(x_{0}\right)=1-x_{0}^{a}, a>0$, decreasing with initial fragment size $x_{0}$. In this model, smaller fragments, once produced, generate (with larger probability) other fragments with smaller sizes, with themselves a larger probability to undergo further splitting and so on. One suspects this fragmentation process to be unstable in that the fragmentation tree is extremely leafy and bushy.

We first derive the fragment size distribution and then study the induced tree structure.

4.1. Unstable fragment size distribution. Under our assumptions, the moment function of the fragment size now satisfies the integral equation

$$
\Phi_{x_{0}}(q)=x_{0}^{q+a}+\left(1-x_{0}^{a}\right) \int_{0}^{1} \Phi_{u x_{0}}(q) \pi(u) d u .
$$

In particular, for $\mathbb{E}\left[X\left(x_{0}\right)\right]=: x\left(x_{0}\right)$, we have

$$
x\left(x_{0}\right)=x_{0}^{a+1}+\left(1-x_{0}^{a}\right) \int_{0}^{1} x\left(u x_{0}\right) \pi(u) d u .
$$

Upon iterating, and using again Lemma 3 of Iserles and Liu (1997), we find the uniqueness of $C^{\infty}$ solutions and

Proposition 4.

(i) We have

$$
x\left(x_{0}\right)=\sum_{m \geq 1}(-1)^{m-1} a_{m} x_{0}^{m a+1},
$$

where

$$
\begin{aligned}
a_{1} & =\frac{1}{1-\phi(a+1)}, \\
a_{m} & =\frac{\prod_{k=1}^{m-1} \phi(1+k a)}{\prod_{k=1}^{m}(1-\phi(1+k a))}, \quad m \geq 2 .
\end{aligned}
$$

(ii) More generally, for all $q>q_{*}=-a$,

$$
\Phi_{x_{0}}(q)=\sum_{m \geq 1}(-1)^{m-1} a_{m}(q) x_{0}^{m a+q},
$$


where

$$
\begin{aligned}
a_{1}(q) & =\frac{1}{1-\phi(q+a)}, \\
a_{m}(q) & =\frac{\prod_{k=1}^{m-1} \phi(q+k a)}{\prod_{k=1}^{m}(1-\phi(q+k a))}, \quad m \geq 2 .
\end{aligned}
$$

Proof. $x\left(x_{0}\right)$ can be searched in the form

$$
x\left(x_{0}\right)=\sum_{m \geq 1}(-1)^{m-1} a_{m} x_{0}^{m a+1},
$$

where the coefficients $a_{m}$ are to be determined. Putting the series expansion into the functional equation which $x\left(x_{0}\right)$ satisfies, we obtain

$$
\sum_{m \geq 1}(-1)^{m-1} a_{m} x_{0}^{m a+1}=x_{0}^{a+1}+\left(1-x_{0}^{a}\right) \sum_{m \geq 1}(-1)^{m-1} a_{m} x_{0}^{m a+1} \phi(m a+1) .
$$

Identifying the terms, we get

$$
\begin{aligned}
a_{1} & =1 /(1-\phi(a+1)), \\
a_{m} & =a_{m} \phi(1+m a)+a_{m-1} \phi(1+(m-1) a), \quad m \geq 2,
\end{aligned}
$$

leading to

$$
a_{m}=\frac{\prod_{k=1}^{m-1} \phi(1+k a)}{\prod_{k=1}^{m}(1-\phi(1+k a))}, \quad m \geq 2 .
$$

Under our assumptions, the series $\sum_{m \geq 1} a_{m} x_{0}^{m a+1}$ with positive terms is always convergent because

$$
\frac{a_{m+1} x_{0}^{(m+1) a+1}}{a_{m} x_{0}^{m a+1}}=\frac{\phi(1+m a)}{1-\phi(1+(m+1) a)} x_{0}^{a} \underset{m \uparrow \infty}{\longrightarrow} 0<1 .
$$

So, $\sum_{m \geq 1}(-1)^{m-1} a_{m} x_{0}^{m a+1}$ is absolutely convergent, hence convergent.

Concerning $\Phi_{x_{0}}(q)$ itself, proceeding similarly, we obtain the solutions displayed in (ii).

Remarks. 1) The moment function $q \mapsto \Phi_{x_{0}}(q)$, defined in (10), is also the Laplace transform of the function $0<t \mapsto f_{x_{0}}\left(e^{-t}\right) e^{-t}$. From the expression of $a_{1}(q)$, it has a dominant singularity at $q_{*}=-a$ which is a simple pole. It follows from elementary singularity analysis (see for example Section 5.2 of Wilf 1994) that $f_{x_{0}}\left(e^{-t}\right) e^{-t} \sim_{t \uparrow \infty} A\left(x_{0}\right) e^{q_{*} t}$. After the change of variable $x=e^{-t}$, we obtain an algebraic divergence $(x \downarrow 0)$ for fragment size density, the scaling exponent of which is $-\left(q_{*}+1\right)$. In other words, we get

$$
f_{x_{0}}(x) \underset{x \downarrow 0}{\sim} A\left(x_{0}\right) x^{-(1-a)} .
$$

It diverges (resp. vanishes) when $a<1$ (resp. $a>1$ ). 
The constant $A\left(x_{0}\right)=\lim _{q \rightarrow q_{*}=-a}\left(q-q_{*}\right) \Phi_{x_{0}}(q)$ is found to be

$$
A\left(x_{0}\right)=\frac{1}{-\phi^{\prime}(0)}\left(1+\sum_{m \geq 1}(-1)^{m} \prod_{k=1}^{m} \frac{\phi((k-1) a)}{1-\phi(k a)} x_{0}^{m a}\right) .
$$

Note that $a$ determines the scaling exponent in the unstable fragmentation model.

2) The law of $X\left(x_{0}\right)$ clearly also has an atom at $x=x_{0}$, now with mass $x_{0}^{a}$ when splitting does not take place. At $q=0$, one can check that $\Phi_{x_{0}}(0)<1$. Therefore $\mathbb{P}\left(X\left(x_{0}\right)=0\right)=1-\Phi_{x_{0}}(0)>0$ : the height $H\left(x_{0}\right)$ of the random path is now infinite with some positive probability.

When $a \uparrow \infty, \Phi_{x_{0}}(q) \rightarrow 0$ for $q>0$; this means that $X\left(x_{0}\right) \rightarrow 0$ in distribution and in probability: as splitting probability tends to 1 , the fragment size tends to zero. When $a \downarrow 0$, the splitting probability tends to 0 and $\Phi_{x_{0}}(q) \sim x_{0}^{q}$.

Finally, the range of $X\left(x_{0}\right)$ is $\left[0, x_{0}\right]$. Define $\tilde{X}\left(x_{0}\right):=X\left(x_{0}\right) / x_{0}$; then the range of $\widetilde{X}\left(x_{0}\right)$ is $[0,1]$, its moment function is

$$
\widetilde{\Phi}_{x_{0}}(q):=\mathbb{E}\left[\widetilde{X}\left(x_{0}\right)^{q}\right]=\sum_{m \geq 1}(-1)^{m-1} a_{m}(q) x_{0}^{m a}
$$

and $\widetilde{X}\left(x_{0}\right)$ is not a scaling quantity $\left(\widetilde{X}\left(x_{0}\right) \stackrel{d}{\neq} x_{0} X_{1}\right)$. When $q=0$, we have $\widetilde{\Phi}_{x_{0}}(q)<1: \widetilde{X}\left(x_{0}\right)=0$ with positive probability. When $q \uparrow \infty, \widetilde{\Phi}_{x_{0}}(q) \rightarrow x_{0}^{a}$, which is the probability not to split: $\widetilde{X}\left(x_{0}\right)$ has an atom at $x=1$.

EXAMPLES.

- Assume $\theta=1, b=2$ and $\phi(q)=(1+q)^{-1}$. If $a=1$, we find

$$
a_{m}(q)=\frac{q+m+1}{\prod_{k=1}^{m}(q+k)} .
$$

Thus, $a_{m}=a_{m}(1)=(m+2) /(m+1)$ !, leading to the average fragment length

$$
x\left(x_{0}\right)=2 x_{0}-1+e^{-x_{0}}\left(1-x_{0}\right) \in\left(0, x_{0}\right) .
$$

The function $x_{0} \mapsto x\left(x_{0}\right)$ is continuous and monotone.

- Assume $a=1 / 2, b=3$ and $\theta=1 / 2$. In this case, we have $\phi(q)=$ $(2 q+1)^{-1}$, and we find

$$
a_{m}(q)=\frac{2 q+m+1}{\prod_{k=1}^{m}(2 q+k)} .
$$

Thus, $a_{m}:=a_{m}(1)=(2 m+6) /(m+2)$ !, leading to the average fragment length

$$
x\left(x_{0}\right)=2+3 x_{0}-4 \sqrt{x_{0}}+2 e^{-\sqrt{x_{0}}}\left(\sqrt{x_{0}}-1\right) \in\left(0, x_{0}\right) .
$$

The function $x_{0} \mapsto x\left(x_{0}\right)$ is continuous and monotone. 
4.2. Number of fragments in the unstable fragmentation tree. We start with the limiting number of leaves (or fragments) in the process, starting with an interval of length $x_{0}$, say $N\left(x_{0}\right)$. First, $N\left(x_{0}\right)<\infty$ with positive probability at least equal to $x_{0}^{a}$ (the probability that $N\left(x_{0}\right)=1$ ). With $N_{k}(\cdot) \stackrel{d}{=} N(\cdot)$, it satisfies

$$
N\left(x_{0}\right) \stackrel{d}{=} \bar{B}\left(x_{0}\right)+B\left(x_{0}\right) \sum_{k=1}^{b} N_{k}\left(U_{k} x_{0}\right) .
$$

Let us consider the expected value $m\left(x_{0}\right):=\mathbb{E}\left(N\left(x_{0}\right)\right)$. If this quantity exists, it fulfills the functional equation

$$
m\left(x_{0}\right)=x_{0}^{a}+b\left(1-x_{0}^{a}\right) \int_{0}^{1} m\left(u x_{0}\right) \pi(u) d u .
$$

Then we have the following result:

Proposition 5. For all $a>0$ and $x_{0} \in(0,1)$ we have $m\left(x_{0}\right)=\infty$.

Proof. Looking for formal unique $C^{\infty}$ solutions of the form

$$
m\left(x_{0}\right)=\sum_{m \geq 1}(-1)^{m-1} b_{m} x_{0}^{m a},
$$

for some unknown sequence $b_{m}, m \geq 1$, we obtain

$$
\begin{aligned}
b_{1} & =\frac{1}{1-b \phi(a)}, \\
b_{m} & =\frac{\prod_{k=1}^{m-1} b \phi(k a)}{\prod_{k=1}^{m}(1-b \phi(k a))}, \quad m \geq 2 .
\end{aligned}
$$

Fix $x_{0} \in(0,1)$. The quantity $m\left(x_{0}\right)$, as a function of the parameter $a>0$, is increasing because the splitting probability $1-x_{0}^{a}$ is now increasing in $a$ for all $x_{0}$. On the other hand, the series representation of $m\left(x_{0}\right)$ shows that $m\left(x_{0}\right)$ diverges for values of $a$ of the form $a=1 / k$ where $k \geq 1$ is any integer.

Thus, $m\left(x_{0}\right)<\infty$ for all $a<a_{\mathrm{c}}$ where $a_{\mathrm{c}}=\inf \left\{a: m\left(x_{0}\right)=\infty\right\}$. We conclude that the sole trivial value of $a$ for which $m\left(x_{0}\right)<\infty$ is $a=0$ (in which case $m\left(x_{0}\right)=1$ since the splitting probability is 0 ). Conversely, for any $a>0, m\left(x_{0}\right)=\infty$.

To conclude, the expected number of leaves in the unstable fragmentation tree is infinite. This result supports (but does not prove) the conjecture that the explosion probability $\mathbb{P}\left(N\left(x_{0}\right)=\infty\right)$ of this model is positive for all $a>0$ and $x_{0} \in(0,1)$. We have not been able to supply a closed form expression for this probability. 
4.3. Partition function. We shall now study the random partition function

$$
Z_{x_{0}}(\beta):=\sum_{i=1}^{N\left(x_{0}\right)} X_{i}\left(x_{0}\right)^{\beta}
$$

with $Z_{x_{0}}(1)=x_{0}$ and $Z_{x_{0}}(0)=N\left(x_{0}\right)$. Let $Z_{x_{0}, k}(\beta), k=1, \ldots, b$, be independent statistical copies of $Z_{x_{0}}(\beta)$. They must solve

$$
Z_{x_{0}}(\beta) \stackrel{d}{=} x_{0}^{\beta} \bar{B}\left(x_{0}\right)+B\left(x_{0}\right) \sum_{k=1}^{b} Z_{U_{k} x_{0}, k}(\beta) .
$$

Let $z_{x_{0}}(\beta):=\mathbb{E}\left(Z_{x_{0}}(\beta)\right)$ be the first moment of $Z_{x_{0}}(\beta)$. Then

$$
z_{x_{0}}(\beta)=x_{0}^{\beta+a}+b\left(1-x_{0}^{a}\right) \int_{0}^{1} z_{u x_{0}}(\beta) \pi(u) d u .
$$

Assume that $a>0$. Then the definition domain of $z_{x_{0}}(\beta)$ is included in $\{\beta: \beta>0\}$ because $z_{x_{0}}(0)=m\left(x_{0}\right)$ diverges for all $a>0$. We obtain the following result:

\section{Proposition 6.}

(i) When $\beta>\beta_{\mathrm{c}}:=\max (0,1-a), z_{x_{0}}(\beta)$ is well defined and

$$
z_{x_{0}}(\beta)=\sum_{m \geq 1}(-1)^{m-1} b_{m}(\beta) x_{0}^{m a+\beta}
$$

is the unique $C^{\infty}$ solution in $x_{0}$ for $(13)$. Here the sequence $b_{m}(\beta)$, $m \geq 1$, is defined by

$$
\begin{aligned}
b_{1}(\beta) & =\frac{1}{1-b \phi(a+\beta)}, \\
b_{m}(\beta) & =b_{1}(\beta) \prod_{k=1}^{m-1} \frac{b \phi(\beta+k a)}{1-b \phi(\beta+(k+1) a)}, \quad m \geq 2 .
\end{aligned}
$$

(ii) Assume $0<a \leq 1$. Then with $\beta_{\mathrm{c}}=1-a \geq 0$ we have: for all $b \geq 2$, there exists $0<C_{a, b}=(1-b \phi(1+a))^{1 / a}<1$ such that for all $x_{0} \in\left(0, C_{a, b}\right]$,

$$
z_{x_{0}}(\beta) \underset{\beta=1-a}{\sim} C_{a}\left(x_{0}\right)\left(\beta-\beta_{\mathrm{c}}\right)^{-1}
$$

for the positive constant

$$
C_{a}\left(x_{0}\right)=\frac{x_{0}}{-b \phi^{\prime}(1)}\left(1-\sum_{m \geq 1}(-1)^{m+1} \prod_{k=1}^{m} \frac{b \phi(1+(k-1) a)}{1-b \phi(1+k a)} x_{0}^{m a}\right) .
$$

Proof. (i) Looking for formal solutions of $z_{x_{0}}(\beta)$ of the form

$$
z_{x_{0}}(\beta)=\sum_{m \geq 1}(-1)^{m-1} b_{m}(\beta) x_{0}^{m a+\beta},
$$


we obtain the coefficients

$$
\begin{aligned}
b_{1}(\beta) & =\frac{1}{1-b \phi(a+\beta)}, \\
b_{m}(\beta) & =\frac{\prod_{k=1}^{m-1} b \phi(\beta+k a)}{\prod_{k=1}^{m}(1-b \phi(\beta+k a))}, \quad m \geq 2 .
\end{aligned}
$$

Clearly, $z_{x_{0}}(\beta)$ diverges for values of $\beta$ of the form $\beta=1-k a$ where $k \geq 1$. Next, for $\beta>0$, the function $z_{x_{0}}(\beta)$ is decreasing in $\beta$. This shows that $z_{x_{0}}(\beta)<\infty$ for $\beta>\beta_{\mathrm{c}}$ where $\beta_{\mathrm{c}}=\sup \left\{\beta>0: z_{x_{0}}(\beta)=\infty\right\}=\max (0,1-a)$.

(ii) Assume $a \leq 1$ and set $\beta_{\mathrm{c}}=1-a \geq 0$. Then it is easy to check that

$$
\begin{aligned}
z_{x_{0}}(\beta) & =x_{0}^{a+\beta} b_{1}(\beta)\left(1+\sum_{m \geq 1}(-1)^{m} \prod_{k=1}^{m} \frac{b \phi(\beta+k a)}{1-b \phi(\beta+(k+1) a)} x_{0}^{m a}\right) \\
& \underset{\beta=1-a}{\sim} C_{a}\left(x_{0}\right)\left(\beta-\beta_{\mathrm{c}}\right)^{-1},
\end{aligned}
$$

where $C_{a}\left(x_{0}\right)$ is given in (ii).

We shall now study the convergence of the function $C_{a}\left(x_{0}\right)$. First, let

$$
A_{m}:=\prod_{k=1}^{m} \frac{b \phi(1+(k-1) a)}{1-b \phi(1+k a)} x_{0}^{m a} .
$$

For $1 \leq k \leq m$, one can check that

$$
0 \leq \frac{b \phi(1+(m-1) a)}{1-b \phi(1+m a)} \leq \frac{b \phi(1+(k-1) a)}{1-b \phi(1+k a)} \leq \frac{1}{1-b \phi(1+a)} .
$$

Therefore,

$$
\left|\sum_{m \geq 1}(-1)^{m+1} A_{m}\right| \leq \sum_{m \geq 1}\left(\frac{x_{0}^{a}}{1-b \phi(1+a)}\right)^{m} .
$$

Thus, for all $a>0$ and $b \geq 2, C_{a}\left(x_{0}\right)$ is absolutely convergent for all $x_{0} \in\left(0, C_{a, b}\right)$, where $0<C_{a, b}=(1-b \phi(1+a))^{1 / a}<1$.

To study the convergence at the point $C_{a, b}$, we shall substitute 1 $b \phi(1+a)$ for $x_{0}^{a}$ in the expression of $C_{a}\left(x_{0}\right)$. We obtain

$$
C_{a}\left(x_{0}\right)=\frac{x_{0}}{-b \phi^{\prime}(1)}\left(1-\sum_{m \geq 1}(-1)^{m+1} B_{m}\right),
$$

with

$$
B_{m}=\prod_{k=1}^{m} \frac{(b \phi(1+(k-1) a))(1-b \phi(1+a))}{1-b \phi(1+k a)}
$$

We get

$$
\frac{B_{m+1}}{B_{m}}=\frac{(b \phi(1+m a))(1-b \phi(1+a))}{1-b \phi(1+(m+1) a)}=: b_{m+1}
$$


with $b \phi(1+m a)<1$ as a result of $1+m a>1$. Further, one can check that

$$
\frac{1-b \phi(1+a)}{1-b \phi(1+(m+1) a)}<1
$$

and so $b_{m+1}<1$. Thus, $B_{m}$ is a decreasing sequence. Next,

$$
\frac{b \phi(1+k a)}{1-b \phi(1+(k+1) a)}<\frac{b \phi(1+(k-1) a)}{1-b \phi(1+k a)}, \quad \forall k \geq 2 .
$$

Consequently,

$$
B_{m} \leq \prod_{k=2}^{m} \frac{b \phi(1+a)(1-b \phi(1+a))}{1-b \phi(1+2 a)}=b_{2}^{m-1},
$$

converging to 0 as $m \uparrow \infty$, because $b_{2}<1$. By the alternating series theorem, $C_{a}\left(x_{0}\right)$ is convergent at $x_{0}=C_{a, b}$. Further, $C_{a, b} \rightarrow_{a \uparrow \infty} 1$ and $C_{a, b} \rightarrow_{a \uparrow 0^{+}} 0$. Since

$$
\begin{aligned}
\left|\sum_{m \geq 1}(-1)^{m+1} \prod_{k=1}^{m} \frac{b \phi(1+(k-1) a)}{1-b \phi(1+k a)} x_{0}^{m a}\right| & =\left|\sum_{m \geq 1}(-1)^{m+1}\left(\frac{x_{0}}{C_{a, b}}\right)^{m a} B_{m}\right| \\
& \leq B_{1}=1,
\end{aligned}
$$

for $0<a \leq 1$ we get $C_{a}\left(x_{0}\right) \geq 0$ for all $x_{0} \in\left(0, C_{a, b}\right]$.

From this study, we obtain the following result

Lemma 7. When $a \leq 1$ and $\sigma(d x):=\mathbb{E} \sum_{i=1}^{N\left(x_{0}\right)} \mathbf{I}\left(X_{i}\left(x_{0}\right) \in d x\right)$, the occupation measure satisfies

$$
\sigma(d x) \underset{x \downarrow 0}{\sim} C_{a}\left(x_{0}\right) x^{-(2-a)} d x,
$$

where $C_{a}\left(x_{0}\right)$ is defined by (14).

Proof. Assume $a \leq 1$. Let $\sigma(d x)$ be the structural measure of the system $\left(X_{i}\left(x_{0}\right) ; i \geq 1\right)$. Then

$$
z_{x_{0}}(\beta)=\int_{0}^{x_{0}} x^{\beta} \sigma(d x) .
$$

From Proposition 6(ii), $z_{x_{0}}(\beta)$ has a simple pole at $\beta_{\mathrm{c}}=1-a$; from singularity analysis, we obtain

$$
\sigma(d x) / d x \underset{x \downarrow 0}{\sim} C_{a}\left(x_{0}\right) x^{-\left(\beta_{c}+1\right)}=C_{a}\left(x_{0}\right) x^{-(2-a)},
$$

where $2-a \in(1,2) \geq 1$.

\section{References}

M. D. Brennan and R. Durrett (1986), Splitting intervals, Ann. Probab. 14, 1024-1036.

M. D. Brennan and R. Durrett (1987), Splitting intervals. II. Limit laws for lengths, Probab. Theory Related Fields 75, 109-127. 
A. F. Filippov (1961), On the distribution of the sizes of particles which undergo splitting, Theory Probab. Appl. 6, 275-294.

T. Huillet (2005), Statistical aspects of random fragmentations, J. Comput. Appl. Math. $181,364-387$.

A. Iserles and Y. Liu (1997), Integro-differential equations and generalized hypergeometric functions, J. Math. Anal. Appl. 208, 404-424.

I. Jeon (2002), Stochastic fragmentation and some sufficient conditions for shattering transition, J. Korean Math. Soc. 39, 543-558.

E. D. McGrady and R. M. Ziff (1987), "Shattering" transition in fragmentation, Phys. Rev. Lett. 58, 892-895.

H. W. Wilf (1994), Generatingfunctionology, 2nd ed., Academic Press, San Diego, CA.

M. Ghorbel

Laboratoire de Physique Théorique et Modélisation

CNRS-UMR 8089 et Université de Cergy-Pontoise

2 Avenue Adolphe Chauvin

95032 Cergy-Pontoise, France

and

Laboratoire d' Analyse, Géometrie et Applications

CNRS-UMR 7539, Institut Galilée

Université de Paris 13

93340 Villetaneuse, France

E-mail: ghorbel@math.univ-paris13.fr

T. Huillet

Laboratoire de Physique Théorique et Modélisation

CNRS-UMR 8089 et Université de Cergy-Pontoise

2 Avenue Adolphe Chauvin

95032 Cergy-Pontoise, France

E-mail: Thierry.Huillet@ptm.u-cergy.fr

Received on 10.7.2005;

revised version on 3.4 .2006 